\title{
Nitric Oxide Metabolites and Asymmetric Dimethylarginine Concentrations in Breast Milk
}

\author{
Hakan ÖZTÜRK' ${ }^{1}$ Esin KOÇ², Taner ÖZGÜRTAŞ̧̧3, Fatih YEŞiLDAL ${ }^{3}$, Ebru KAZANCI², Meltem AKSU², \\ K. Ebru ÖZCAN², İbrahim M. HİRFANOĞLU ${ }^{2}$, Esra ÖNAL ${ }^{2}$, Canan TÜRKYILMAZ², Ebru ERGENEKON², \\ Yıldız ATALAY2
}

Ankara, Turkey

ABSTRACT

OBJECTIVE: Nitric oxide plays a preventive role in the development of necrotizing enterocolitis. Oral nitrite and nitrate intake has gained importance with the discovery of the conversion of nitrite to nitric oxide in acidic medium out of the synthesis of nitric oxide from L-arginine. Objective of this study was to examine the breast milk concentrations of nitric oxide and asymmetric dimethylarginine which is a competitive inhibitor of nitric oxide and to compare these concentrations in terms of gestational age and maturity of breast milk.

STUDY DESIGN: Forty-one women were included in the study. Milk samples were collected from 3 groups of mothers as term, late preterm and preterm on the postpartum days 3,7 and 28 .

RESULTS: When breast milk concentrations of nitric oxide were compared according to the postnatal day of the milk independently from gestational age; nitric oxide concentration was higher in the colostrum than in the transition milk and mature milk $(p=0,035 ; p=0,001$; respectively). For the comparison of asymmetric dimethylarginine concentrations among these groups and days; no statistically significant difference was observed in terms of gestational age and maturity of the milk ( $p=0.865, p=0.115$; respectively).

CONCLUSION: The highest nitric oxide concentration was found in the colostrum, suggesting that colostrum is a valuable food for newborns. Plasma concentrations of asymmetric dimethylarginine were negatively correlated with nitric oxide and did not show a correlation with breast milk, suggesting that asymmetric dimethylargininedoesn't make nitric oxide inhibition in breast milk.

Keywords: Breast milk, Nitric oxide, Asymmetric dimethylarginine

Gynecol Obstet Reprod Med 2017;23(1):41-44

\section{Introduction}

Nitric oxide (NO) is a potent vasodilator agent. Following Moncada et al showed in 1987 that endothelium-derived relaxing factor is actually NO, this agent has been subject to numerous studies (1). It has been discovered that in addition to being a vasodilator agent, NO is an anti-inflammatory, antiox-

\footnotetext{
${ }^{1}$ Department of Pediatrics, and 2 Department of Pediatrics Division of Neonatology Gazi University Medical Faculty, Ankara

${ }^{3}$ Gulhane Military Medical Academy, Department of Biochemistry and Clinical Biochemistry, Ankara
}

Address of Correspondence: Hakan Öztürk

Gazi University Medical Faculty

Department of Pediatrics Ankara, Turkey

hakan.ozturk@gazi.edu.tr

Submitted for Publication: $\quad$ 18.01.2017

Accepted for Publication: $\quad$ 27.03.2017

\begin{tabular}{|c|c|}
\hline & Access this article online \\
\hline $\begin{array}{c}\text { Quick Response Code: } \\
\text { Website: www.gorm.com.tr }\end{array}$ \\
\cline { 2 - 2 }
\end{tabular}

How to cite this article: Öztürk H. Koç E. Özgürtaș T. Yeşildal F. Kazancı E. Aksu M. et al. Nitric Oxide Metabolites and Asymmetric Dimethylarginine Concentrations in Breast Milk. Gynecol Obstet Reprod Med 2017;23(1):41-44 idant, antiaggregant and antiatherosclerotic agent as well as it functions as a signal molecule in the intercellular communication. It is synthesized from L-arginine through nitric oxide synthase (NOS) enzyme. Then it is oxidized to nitrite and nitrate. It has been believed that nitrite and nitrate were end products of NO which having no bioactivity. However, in two independent studies conducted in 1994, it was discovered that nitrite converted to NO in acidic medium $(2,3)$. Asymmetric dimethylarginine (ADMA) is a competitive inhibitor of NOS. It has been demonstrated in many studies that concentration of NO decreased in the cases of increased concentration of ADMA. ADMA is recognized as an independent factor in cardiovascular diseases, chronic renal and hepatic diseases and critically ill patients (4-6).

Necrotizing enterocolitis (NEC) is characterized by a decreased gastrointestinal blood flow, thinning and perforation in the mucus barrier and pathogenic bacteria invasion. Pathogenesis of NEC is not fully understood. However, the accused factors include prematurity, hyperosmolar feeding, hypoxic ischemic injury, insufficient mucosal defense, irregularity in the bowel blood flow and bacterial colonization (7). NO has effects on many factors that are considered to play a role in development of NEC (8). The balance between NO and 
endotelin-1 is very important in the regulation of bowel blood flow. This balance shifts to the vasoconstriction side in the cases of hypoxia, endothelium dysfunction and impaired blood flow which leads to development of NEC (9). Whereas NO increases mucosal blood flow and mucus secretion, undertaking a protective role against NEC. One of the most important factors in the pathogenesis of NEC is hypoxic ischemic injury. The body directs the blood as a reflex to the vital organs such as the heart and brain in cases that disturbs the circulation such as hypoxia, acidosis, hypotension and hypothermia in newborns and ischemia may occur on development of severe vasoconstriction in the vessels that supplies the bowel (10). Following ischemia, inflammatory mediators are secreted, increasing mucosal damage and accelerating the development of NEC (11). NO is protective against hypoxic ischemic injury owing its antioxidant and anti-inflammatory effects $(12,13)$.

Orally taken nitrite and nitrate are resources for gastric NO. Primarily nitrate converts to nitrite through nitrate reductase enzyme by oral flora bacteria. Then nitrite converts to NO in the acidic medium of the stomach independently from NOS (2). However, since majority of newborns is fed with breast milk exclusively, their only resource of nitrite and nitrate is breast milk.

This study was designed to examine breast milk concentrations of NO which is known to play a role in the pathogenesis of NEC, whether these concentrations differ in terms of the gestational and postnatal age, and if ADMA which is an inhibitor of NOS was correlated with NO.

\section{Material and Method}

We included total 41 mothers of 14 preterm, 13 late preterm and 14 term newborns in the study. A total of three samples was collected from the mothers on the postpartum days 3, 7 and 28. Breast milks were collected on the day 3 were termed as colostrum, day 7 as transition milk and day 28 as mature milk. Infants born in the $34^{\text {th }}$ gestational week and earlier were considered as preterm, those born between the $34^{\text {th }}$ and $37^{\text {th }}$ weeks as late preterm and newborns born in the $37^{\text {th }}$ week and over were considered as term. Mothers emptied their single breast at mornings (between 9-10 am) in the specified days with the help of a vacuum pump (Medela Mini Electric), shaked the milk and put the milk of $4 \mathrm{cc}$ in a glass tube using an injector and delivered it at $+4{ }^{\circ} \mathrm{C}$ to the laboratory with ice accumulators at the latest 12 hours. Mothers emptied their single breasts fully and mixed the milk because fore and hindmilk contents can differ. After the samples were delivered to the laboratory, they were centrifuged at 4000 ro- tates/minute at $+4{ }^{\circ} \mathrm{C}$ for 10 minutes. Layer of fat in the surface was crossed through a thin injector and supernatant partition was collected. Supernatant partition was put into the Eppendorf tubes and kept at $-80{ }^{\circ} \mathrm{C}$ until the analysis. Diazotization method was used for the measurement of nitrite and nitrate in the breast milk. According to this method, nitrate primarily converts to nitrite through nitrate reductase. Then nitrite and sulfanilamide reacts with N-ethylenediamine in the acidic medium, creating a pink colored complex. The method is based on measurement of the absorbance of this complex at $540 \mathrm{~nm}$ wavelength. This is known as the Griess reaction. Device used in the absorbance measurement was Model ELx 800 Bioelisa Microplate Reader Biokit, Spain. Measurement of ADMA concentration in breast milk was performed using high performance liquid chromatography (HPLC) method. The HPLC device used for this was Shimadzu LC, Japan. Data obtained were evaluated using SPSS 16.0 packet software. Statistical analyses were performed through Friedman Test, Wilcoxon Signed Rank Test and Kruskal Wallis H Test. This study was approved by Gazi University, Medical Faculty, Clinical Research Ethics Committee. Mothers included in the study were verbally informed and their consents were received.

\section{Results}

Mean ages and gestational ages of the mothers are given in the table 1 .

When breast milk concentrations of NO were compared independently from the gestational age; NO concentrations in the colostrum were found to be higher than in the transition and mature milks ( $p=0,035 ; p=0,001$; respectively). NO concentration in the transition milk was higher compared to the mature milk, although the difference was not statistically significant $(p=0,110)$. Change of the NO concentrations in breast milk according to the postnatal days is shown in the figure 1 .

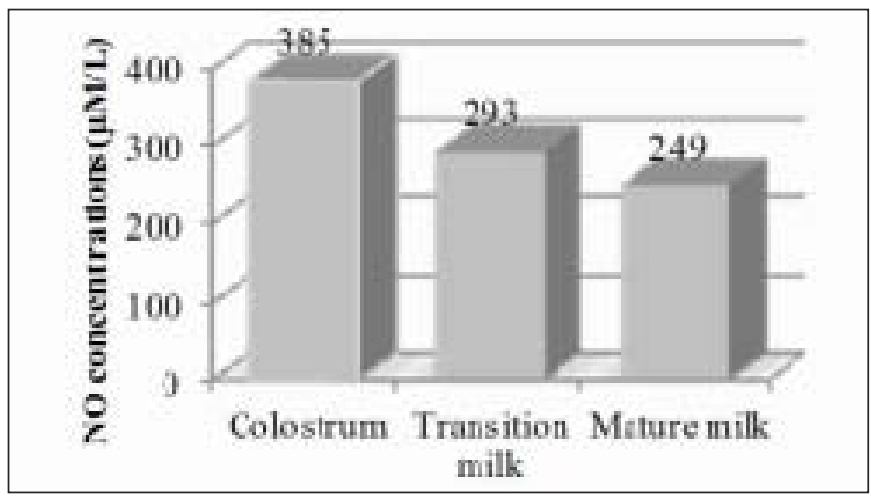

Figure 1: Change of the NO concentrations in breast milk according to the postnatal days $(n=41)$

Table 1: Demographic characteristics of the mothers included in the study

\begin{tabular}{lllll}
\hline & Preterm $(n=14)$ & Late Preterm $(n=13)$ & Term $(n=14)$ & All $(n=41)$ \\
\hline Mother's age (year) & $28,5 \pm 4,8$ & $29,6 \pm 3,9$ & $30,3 \pm 4,2$ & $29,5 \pm 4,2$ \\
Gestational age (week) & $31 \pm 2,2$ & $35,7 \pm 0,8$ & $39 \pm 0,7$ & $35,1 \pm 3,7$ \\
\hline
\end{tabular}

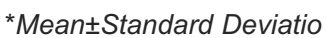


When NO concentrations were compared in terms of gestational ages, no statistically significant difference was observed among the preterm, late preterm and term groups $(p=0,429)$. In addition, no statistically significant difference was found among the group in ADMA concentrations in terms of the postnatal days and gestational ages $(p=0,115 ; p=0,865$; respectively).

\section{Discussion}

In this study, when breast milk NO concentrations were examined; the highest concentration was found in the colostrum, indicating that colostrum is a valuable food for newborns. NO concentrations in breast milk gradually decrease in the forthcoming postnatal days. Given the role which NO plays in the pathogenesis of NEC and newborns often diagnosed with NEC after the first week of life, suggests that although insufficient NO intake is not the only reason, it may contribute to the development of NEC. There are two studies in which L-arginine which is precursor of NO was given to the newborns, considering NO is protective against NEC $(14,15)$. In these studies, a total of 115 preterm newborns was administered intravenous or enteral L-arginine $1.5 \mathrm{mmol} / \mathrm{kg} /$ day and 120 preterm newborns were given placebo. It was found in a meta-analysis which included these two studies that prophylactic administration of L-arginine decreased the incidence of stage 2-3 NEC by 59\% (16). This result supports that insufficient NO intake may have a role in the development of NEC.

Plasma concentrations of nitrite and nitrate is closely related to diet. It has been found in a conducted study that, plasma concentrations of nitrite and nitrate were raised by 2 to 3 folds in those fed with vegetables that are known to have high nitrate content (17). However, oral nitrate intake by newborns has some disadvantages in terms of conversion of nitrate to NO. The first disadvantage for newborns is the lack of oral flora bacteria that convert nitrate to nitrite. It is known that, nitrate reductase activity which was examined in the saliva of newborns is not measurable in the first 5 days of life and its concentration in the weeks 2 to 8 is about only $10 \%$ of that measured in adults (18). The second disadvantage is during conversion of nitrite which reaches to the stomach to NO. This conversion requires an acidic medium. Newborns have a higher stomach $\mathrm{pH}$ than adults (19). However, in order to overcome this disadvantage oral administration of the reductive agents such as vitamin $\mathrm{C}$ or polyphenol increases conversion of nitrite to NO (20). Another disadvantage for the newborns is that amount of nitrite and nitrate taken is rather lower than adults. In a study from the literature on this subject, an average newborn consumes $0,0007 \mathrm{mg} / \mathrm{kg} /$ day nitrite and 0,12 $\mathrm{mg} / \mathrm{kg} /$ day nitrate when it was fed with $150 \mathrm{cc} / \mathrm{kg} /$ day breast milk. When these values are compared with those of an adult; a newborn consumes $0.6 \%$ nitrite and $5 \%$ nitrate of those of consumed by adults. This difference is further increased in the newborns who is parenterally fed (21).
Incidence of NEC increases in newborns as the gestational age decreases. Based on this, the question arises about "is the concentration of NO in breast milk is lower in premature babies?". There is only one study in the literature comparing preterm and term groups in terms of breast milk of NO. In this study, breast milk concentrations of $\mathrm{NO}$ in preterms was found to be lower than in terms (21). However in our study, despite late preterms who were born between the 34 and 37 gestational weeks were accepted as a separate group, unlike that study no statistically significant difference was found between the groups when breast milk concentrations of NO were compared in terms of the gestational ages $(p=0,429)$. Again, in that study, likewise our study concentrations of nitrite and nitrate which were high in the colostrum were found to decrease in the forthcoming postnatal days.

Asymmetric dimethylarginine is competitive inhibitor of NOS which realizes NO synthesis from L-arginine. Since plasma concentration of ADMA is increased in many diseases, it has been introduced as a new marker in terms of the course of disease and mortality $(22,23)$. In a study conducted to define the correlation between NEC and ADMA, plasma concentrations of arginine were found to be lower in the preterms who developed NEC as expected. However, contrary to expectations plasma concentrations of ADMA were also found to be lower in the preterms who developed NEC. Low plasma concentrations of ADMA were thought to be resulted from a compensatory mechanism (24). We studied ADMA in breast milk for the first time in the literature in order to define whether it is correlated with NO. However, in previous studies, plasma concentrations of ADMA were negatively correlated with $\mathrm{NO}$ and did not show a correlation with breast milk, suggesting that ADMA doesn't make NO inhibition in breast milk. When breast milk concentrations of ADMA were compared in terms of gestational ages and postnatal days; no statistically significant difference was found among the groups ( $p=0,865 ; p=0,115$; respectively). ADMA concentrations obtained from the breast milks are close to the plasma concentrations of ADMA from the other studies. Resource of ADMA identified in breast milk is unknown. It can only be speculated that it may be secreted from the mammary gland or transported to the breast milk from the plasma.

Limiting factor of our study was small number of the mothers included. In addition, dietary habits of mothers may cause difference in the concentrations of nitrite and nitrate. However, it was found in a study that change in amount of nitrite and nitrate in diet of the mothers caused change of the plasma concentrations of nitrite and nitrate, while this change had not any effect on breast milk concentrations (25). Therefore, no diet rich or poor in nitrite and nitrate was applied in mothers. NO is difficult to be directly measured because of very short life. Instead, nitrite and nitrate that are end products of NO are used as the markers. According to the technique we used for $\mathrm{NO}$ measure, first nitrate is converted to 
nitrite, then total nitrite is measured. Thus, we have not the chance to separately measure nitrite and nitrate values.

In conclusion, newborns receive limited amounts of nitrite and nitrate from breast milk. NO has a role in the pathogenesis of NEC, suggesting that oral administration of nitrite in preterm newborns may be protective against NEC. However, further studies are needed to define efficiency and to examine side effects such as methemoglobinemia.

\section{References}

1. Marsh N, Marsh A. A short history of nitroglycerine and nitric oxide in pharmacology and physiology. Clin Exp Pharmacol Physiol 2000;27(4):313-9.

2. Benjamin N, O'Driscoll F, Dougall H, Duncan C, Smith L, Golden M, et al. Stomach NO synthesis. Nature 1994; 368(6471):502.

3. Lundberg JO, Weitzberg E, Lundberg JM, Alving K. Intragastric nitric oxide production in humans: measurements in expelled air. Gut 1994;35(11):1543-6.

4. Boger RH, Ron ES. L-Arginine improves vascular function by overcoming deleterious effects of ADMA, a novel cardiovascular risk factor. Altern Med Rev 2005;10(1): 14-23.

5. Siroen MP, van der Sijp JR, Teerlink T, van Schaik C, Nijveldt RJ, van Leeuwen PA. The human liver clears both asymmetric and symmetric dimethylarginine. Hepatology 2005;41(3):559-65.

6. Nijveldt RJ, Teerlink T, Van Der Hoven B, Siroen MP, Kuik DJ, Rauwerda JA, et al. Asymmetrical dimethylarginine (ADMA) in critically ill patients: high plasma ADMA concentration is an independent risk factor of ICU mortality. Clin Nutr 2003;22(1):23-30.

7. Kliegman RM, Walker WA, Yolken RH. Necrotizing enterocolitis: research agenda for a disease of unknown etiology and pathogenesis. Pediatr Res 1993;34(6):701-8.

8. Jones JA, Hopper AO, Power GG, Blood AB. Dietary intake and bio-activation of nitrite and nitrate in newborn infants. Pediatr Res 2015;77(1-2):173-81.

9. Mawji IA, Marsden PA. Perturbations in paracrine control of the circulation: role of the endothelial-derived vasomediators, endothelin-1 and nitric oxide. Microsc Res Tech 2003;60(1):46-58.

10. Neu J. Necrotizing enterocolitis: the search for a unifying pathogenic theory leading to prevention. Pediatr Clin North Am 1996;43(2):409-32.

11. Ford HR, Sorrells DL, Knisely AS. Inflammatory cytokines, nitric oxide, and necrotizing enterocolitis. Semin Pediatr Surg 1996;5(3):155-9.

12. Brown GC, Borutaite V. Nitric oxide inhibition of mitochondrial respiration and its role in cell death. Free Radic Biol Med 2002;33(11):1440-50.
13. Rodriguez-Pena A, Garcia-Criado FJ, Eleno N, Arevalo M, Lopez-Novoa JM. Intrarenal administration of molsidomine, a molecule releasing nitric oxide, reduces renal ischemia-reperfusion injury in rats. Am $\mathrm{J}$ Transplant 2004;4(10):1605-13.

14. Amin HJ, Zamora SA, McMillan DD, Fick GH, Butzner JD, Parsons HG, et al. Arginine supplementation prevents necrotizing enterocolitis in the premature infant. J Pediatr 2002;140(4):425-31.

15. Polycarpou E, Zachaki S, Tsolia M, Papaevangelou V, Polycarpou N, Briana DD, et al. Enteral L-arginine supplementation for prevention of necrotizing enterocolitis in very low birth weight neonates: a double-blind randomized pilot study of efficacy and safety. JPEN J Parenter Enteral Nutr 2013;37(5):617-22.

16. Mitchell K, Lyttle A, Amin H, Shaireen H, Robertson HL, Lodha AK. Arginine supplementation in prevention of necrotizing enterocolitis in the premature infant: an updated systematic review. BMC Pediatr 2014;14:226.

17. Ashworth A, Mitchell K, Blackwell JR, Vanhatalo A, Jones AM. High-nitrate vegetable diet increases plasma nitrate and nitrite concentrations and reduces blood pressure in healthy women. Public Health Nutr 2015:1-10.

18. Kanady JA, Aruni AW, Ninnis JR, Hopper AO, Blood JD, Byrd BL, et al. Nitrate reductase activity of bacteria in saliva of term and preterm infants. Nitric Oxide 2012;27(4):193-200.

19. Miclat NN, Hodgkinson R, Marx GF. Neonatal gastric pH. Anesth Analg 1978;57(1):98-101.

20. Gago B, Lundberg JO, Barbosa RM, Laranjinha J. Red wine-dependent reduction of nitrite to nitric oxide in the stomach. Free Radic Biol Med 2007;43(9):1233-42.

21. Jones JA, Ninnis JR, Hopper AO, Ibrahim Y, Merritt TA, Wan KW, et al. Nitrite and nitrate concentrations and metabolism in breast milk, infant formula, and parenteral nutrition. JPEN J Parenter Enteral Nutr 2014;38(7):856-66.

22. Lu TM, Ding YA, Lin SJ, Lee WS, Tai HC. Plasma levels of asymmetrical dimethylarginine and adverse cardiovascular events after percutaneous coronary intervention. Eur Heart J 2003;24(21):1912-9.

23. Ravani P, Tripepi G, Malberti F, Testa S, Mallamaci F, Zoccali C. Asymmetrical dimethylarginine predicts progression to dialysis and death in patients with chronic kidney disease: a competing risks modeling approach. J Am Soc Nephrol 2005;16(8):2449-55.

24. Richir MC, Siroen MP, van Elburg RM, Fetter WP, Quik F, Nijveldt RJ, et al. Low plasma concentrations of arginine and asymmetric dimethylarginine in premature infants with necrotizing enterocolitis. Br J Nutr 2007;97 (5):906-11

25. Dusdieker LB, Stumbo PJ, Kross BC, Dungy CI. Does increased nitrate ingestion elevate nitrate levels in human milk? Arch Pediatr Adolesc Med 1996;150(3):311-4. 